\section{Brain projects need stronger foundation}

The US BRAIN Initiative and the European Commission's Human Brain Project might be more usefully compared to former US President Richard Nixon's 'War on Cancer' than to the Human Genome Project (Nature 499, 253, 272-274; 2013).

Despite hundreds of millions of dollars being spent after Nixon's 1971 National Cancer Act, the 'war' is still far from over. Back then, cancer scientist Sol Spiegelman remarked: "An all-out effort at this time would be like trying to land a man on the Moon without knowing Newton's laws of gravity."

The same might be said of these huge brain-mapping ventures: we have not yet cracked the neural code, and we have only the most rudimentary understanding of the signature nonlinear dynamics of brain function.

A sound understanding of underlying scientific principles was essential to successful megaprojects such as the Moon landing and the Manhattan Project. Without this, brain-mapping efforts may be premature the equivalent of mapping the structure of snowflakes, while diverting efforts away from understanding the scientific principles that generate them.

The Human Genome Project was of immense benefit to science, but in and of itself it was a feat of engineering. It is unlikely that the project will provide an appropriate blueprint for perhaps the biggest mystery the human brain has ever pondered.

Dean Buonomano Brain Research Institute, University of California, Los Angeles, USA. dbuono@ucla.edu

\section{Step up funding to halt forensic folly}

Forensic science faces challenges in many countries besides the United Kingdom (Nature 500, 5; 2013), particularly in terms of its research funding and culture. If the world's leading economies want science and justice to support each other more effectively and thus allow courts to make informed decisions, forensic science needs to be adequately funded.

Leading funding organizations tend to favour transformational over applied research such as forensic science. Forensic research consequently misses out, even in this age of 'impact' rating.

Genuine breakthroughs in forensic science depend on advances in basic disciplines such as analytical chemistry, molecular biology and biochemistry. But limited funds mean that forensic science has all too often had to depend on the transfer of technology from other fields, combined with statistical, anecdotal or casestudy-based evidence.

Making inferences from single-event observations is poor scientific practice, building a 'house of cards' that can lead to the wrong verdict in court. This may be how case law is formed, but it is not a route to robust science.

If it is to contribute effectively to criminal proceedings, forensic research must be conducted by well-resourced and properly trained scientists.

Mark Tibbett National Soil Resources Institute, Cranfield University, Cranfield, UK. mark.tibbett@cranfield.ac.uk

\section{Science luminaries are often religious}

Young Earth creationists are easy to lampoon (see G. Branch Nature 500, 149; 2013). However, using reasoned arguments might hold more sway with the US creationist movement.

PZ Myers, author of The Happy Atheist (which Branch reviewed), should remember that the majority of those who helped to establish the disciplines that we now practise as modern science were religious believers, including Nicolaus Copernicus, Rene Descartes, Blaise Pascal, Robert Boyle, Isaac Newton, Carl Linnaeus, Edward Jenner, Michael Faraday, Charles Babbage, Joseph Lister, William Thomson and Arthur Stanley Eddington - to name but a few, and excluding a long list of contemporary names. Half of the 10 most influential scientists of the past 350 years chosen for the Royal Society's commemorative stamps in 2010 were religious believers.

Robert White University of Cambridge, UK.

George Ellis University of Cape Town, South Africa.

Denis Alexander Faraday Institute for Science and Religion, St Edmund's College, Cambridge, UK. dra24@hermes.cam.ac.uk

\section{Temper Italy's strict lab-animal law}

The approval in July of a law on animal experimentation in Italy could irreversibly jeopardize the country's medical research, particularly in drug development, and exclude Italy's scientists from international funding (see Nature 499, 258-259; 2013; and Nature http://doi.org/nk3; 2013).

Initially intended to replace a European Union directive that regulates the use of laboratory animals with sensible cautionary guidelines, the draft law was repeatedly amended to satisfy antivivisectionists. The law now forbids xenotransplantation (a widely used method in which human tumour cells are implanted into mice to test cancer therapies) and bans the use of animals for studying drug abuse.

A government initiative is urgently needed to modify the regulations while maintaining rigorous and humane animal experimentation.

Livio Trusolino, Andrea

Bertotti University of Turin School of Medicine; and Institute for Cancer Research and Treatment, Candiolo, Turin, Italy.

livio.trusolino@ircc.it

\section{Ferret H7N9 flu model questioned}

We question the relevance to human pandemics of studies designed to investigate transmissibility of the avian influenza $A(H 7 N 9)$ virus between ferrets (M. Richard et al. Nature http://doi.org/njc; 2013; see also R. A. M. Fouchier et al. Nature 500, 150-151; 2013).

Earlier studies have demonstrated ferret transmission of wild-type A(H7N9) virus (H. Zhu et al. Science 341, 183-186; 2013; and Q. Zhang et al. Science 341, 410-414; 2013). However, the results are in striking contrast with the evidence that human-to-human transmission is extremely infrequent.

The discrepancy between ferret and human transmission in some strains undermines the purported value of 'gain-offunction' (GOF) experiments, which track genetically modified variants of A(H7N9). Yet these studies and a proposal to do GOF experiments (R. A. M. Fouchier et al. Nature 500, 150$151 ; 2013$ ) have been published without seriously questioning the relevance of the ferret model.

A naturally occurring virus could differ subtly from any produced experimentally. In that case, human transmissibility, antigenicity and drug resistance would need to be assessed by studies of the actual strain of virus, and data from GOF experiments could in fact be misleading.

The irony of GOF studies is that these results are likely to be useful for public health only if the pandemic arises from a lab accident, as many fear could happen if this work proliferates. Marc Lipsitch ${ }^{\star}$ Harvard School of Public Health, Boston, Massachusetts, USA. mlipsitc@hsph.harvard.edu ${ }^{\star}$ On behalf of 4 co-authors. Two authors declare competing financial interests: see go.nature. com/eco2zz for details and for full author list. 